\title{
Technology of infrared radiation polarizer
}

\author{
Valentin G. Kolobrodov ${ }^{\mathrm{a}}$, Grygorij S. Tymchik ${ }^{\mathrm{a}}$, Volodymyr I. Mykytenko ${ }^{\mathrm{a}}$, \\ Anatolii M. Kylivnyk ${ }^{\mathrm{b}}$, Ryszard Romaniuk ${ }^{\mathrm{c}}$, Piotr Kisała ${ }^{\mathrm{d}}$, Ainur Kozbakova ${ }^{\mathrm{e}, \mathrm{f}}$, Bakhyt Yeraliyeva ${ }^{\mathrm{g}}$ \\ ${ }^{a}$ National Technical University of Ukraine "Igor Sikorsky Kyiv Polytechnic Institute"; binnytsia \\ State Pedagogical University named after M. Kotsiubynskyi; ${ }^{c}$ Institute of Electronic Systems, \\ Warsaw University of Technology, Warsaw, Poland; ${ }^{\mathrm{d}}$ Lublin University of Technology, Lublin, \\ Poland; 'Institute of Information and Computational Technologies CS MES RK, Almaty,

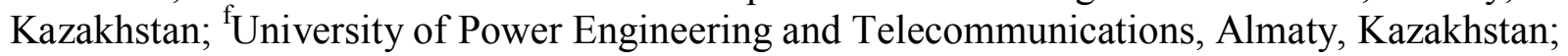 \\ ${ }^{\mathrm{g}}$ Taraz State University after M.Kh.Dulaty, Taraz, Kazakhstan
}

\begin{abstract}
Among the various characteristics of infrared radiation, the degree of polarization is not often used in radiation analysis. The main reason is that polarization is less informative characteristic compared to others for most practical tasks. Also obtaining polarized radiation in infrared spectrum is relative complex and expensive act. In some cases, such as remote sensing, the improvement of spatial, radiometric and spectral resolution approaches it's physical limit. It becomes relevant to obtain additional information of a different nature, such as polarization information. Modern infrared radiation polarizers based on diffraction gratings are quite expensive. The article explores the possibility of creating infrared polarizers based on a planeparallel plate, to which radiation falls at an Brewster angle. It is shown that the polarizer operating on transmittance will be more efficient than reflecting radiation polarizer, since it does not deviate the optical axis by a significant angle. Such a polarizer provides a polarization degree of $90 \%$ and a transmittance of about $50 \%$.
\end{abstract}

Keywords: polarization, infrared radiation, Brewster angle, linear polarization

\section{INTRODUCTION}

Polarization devices are widely used in high-precision measuring systems, coherent optical systems of various purposes, etc. ${ }^{1,2,3,4}$. The main element in such systems is polarizer that convert natural radiation into linear polarized radiation. Two optical phenomena are used in the visible spectral range polarizers ${ }^{5,6}$ : the reflection of light from the surface when the angle of incidence is equal to the Brewster angle; double refraction, which results in two rays linearly polarized in mutually perpendicular planes. The first phenomenon is used little because of significant energy losses.

Usage of polarized infrared radiation in devices and systems of various purposes significantly extends their scope of application $7,8,9,10$. The main problem with the creation of infrared polarization devices is to obtain plane-polarized radiation. The use of double refraction is problematic, since the anisotropic crystals inherent in double refraction are practically absent for mid-infrared spectral range of $8-14 \mu \mathrm{m}$. The most common infrared polarizers use onedimensional transmitting amplitude diffraction grating - ruled or holographic wire grid structures ${ }^{11}$. Polarization efficiency depends on the grid spacing formed among the wires and on the transmittance of substrate. The smaller is spacing the better is polarizer's performance. The spacing between lines is small, typically of $0.12 \mu \mathrm{m}$ in size and with period of $0.25 \mu \mathrm{m}$. Such grating is technologically difficult to make and so it is quite expensive. That is why there is a need to create a cheap polarizer that can be used in laboratories to study IR polarization systems ${ }^{12,13}$.

The purpose of this work is to investigate the feasibility of applying Brewster's law to create IR polarizers.

\section{THE PHYSICAL BASIS OF POLARIZATION}

Let's consider a plane monochromatic wave that falls at an angle $\varepsilon_{1}$ to the boundary of dielectric and semiconductor with refractive indices $n_{1}$ and $n_{2}$ (Figure 1). Part of the radiation is reflected and part is refracted. The first medium is air and second medium is germanium plate, which is transparent in the infrared region of $8-14 \mu \mathrm{m}$. The plate is well polished and therefore a mirror reflection of radiation occurs on its surface.

*e-mail: deanpb@kpi.ua

Optical Fibers and Their Applications 2020, edited by Ryszard S. Romaniuk, Jan Dorosz, Proc. of SPIE Vol. 11456, 1145609 - (c) 2020 SPIE CCC code: $0277-786 \mathrm{X} / 20 / \$ 21 \cdot$ doi: $10.1117 / 12.2569786$ 
Since the reflection and refraction causes partially polarizing of the rays, it is quite difficult to solve the problem of calculating the reflection and transmittance coefficients directly for natural light. To simplify the solution of the problem, lets consider a model of natural light, in which its electrical vector $\vec{E}_{n}$ is represented as the sum of two waves. The waves are linearly polarized in two mutually perpendicular planes and have the same intensity and pass in the direction of natural light (Figure 2). Mathematically, this can be represented as a relation

$$
\vec{E}_{n}=\vec{E}_{\|}+\vec{E}_{\perp} ; I_{n}=I_{\|}+I_{\perp} ; I_{\|}=I_{\perp}=0.5 I_{n}
$$

where $\vec{E}_{\|}=\vec{E}_{p}$ is electrical vector of the wave which plane of polarization is parallel to the plane of incident beam, $\vec{E}_{\perp}=\vec{E}_{s}$ is electrical vector of the wave which plane of polarization is perpendicular to the plane of incidence and $I_{n}, I_{\|}, I_{\perp}$ are intensities of waves $\vec{E}_{n}, \vec{E}_{\|}, \vec{E}_{\perp}$ respectively.

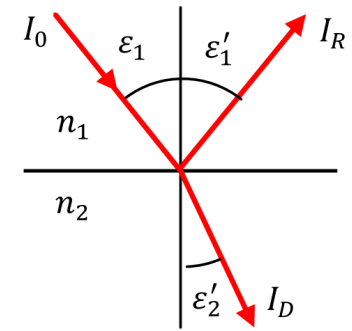

Figure 1. Reflection and refraction of light

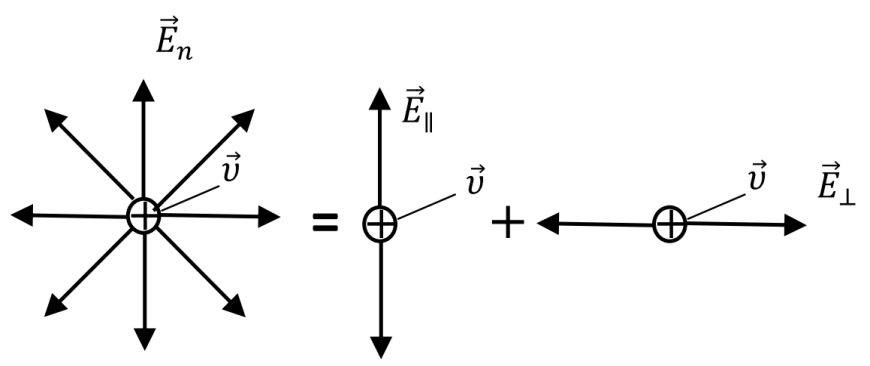

Figure 2. Model of natural radiation

The energy reflection coefficient is calculated by Fresnel formulas ${ }^{3,4}$ :

1. when the light incidence is at a normal angle

$$
R=\frac{I_{R}}{I_{0}}=\left(\frac{E_{R 0}}{E_{0}}\right)^{2}=\left(\frac{n_{1}-n_{2}}{n_{1}+n_{2}}\right)^{2},
$$

2. when the light incidence is at angle of $\varepsilon_{1}$

$$
R=\frac{1}{2}\left(R_{\|}+R_{\perp}\right)=\frac{1}{2}\left(R_{p}+R_{s}\right)=\frac{1}{2}\left\{\left[\frac{\operatorname{tg}\left(\varepsilon_{1}-\varepsilon_{2}^{\prime}\right)}{\operatorname{tg}\left(\varepsilon_{1}+\varepsilon_{2}^{\prime}\right)}\right]^{2}+\left[\frac{\sin \left(\varepsilon_{1}-\varepsilon_{2}^{\prime}\right)}{\sin \left(\varepsilon_{1}+\varepsilon_{2}^{\prime}\right)}\right]^{2}\right\},
$$

where $\varepsilon_{2}^{\prime}$ is the angle of refraction determined by Snell-Descartes' law

$$
\frac{n_{2}}{n_{1}}=\frac{\sin \varepsilon_{1}}{\sin \varepsilon_{2}^{\prime}} .
$$

We have $n_{1}=1$ and $n_{2}=4.0$ for a germanium plate in air. Then the reflection coefficient from the plate in the normal fall according to (2) is equal to $R=\left(\frac{1-4}{1+4}\right)^{2}=0.36$. When the natural beam falls at an angle $\varepsilon_{1}$, the refraction angle $\varepsilon_{2}^{\prime}$ is calculated according to (4) by the formula

$$
\varepsilon_{2}^{\prime}=\arcsin \left(\frac{1}{4} \sin \varepsilon_{1}\right) .
$$

Figure 3 shows the graphs of the dependence of the total coefficient $R$ and the partial energy coefficients $R_{p}$ and $R_{s}$ of reflection on the incidence angle $\varepsilon_{1}$. At the incidence angle $\varepsilon_{1}=0^{\circ}$, the refraction angle is $\varepsilon_{2}^{\prime}=0^{\circ}$. This leads to the uncertainty of the function (3). In this regard, we use the approximations for small angles $\alpha: \operatorname{tg} \alpha \approx \alpha, \sin \alpha \approx \alpha$, and get functions (4) and (3) as

$$
\frac{n_{2}}{n_{1}}=\frac{\sin \varepsilon_{1}}{\sin \varepsilon_{2}^{\prime}} \approx \frac{\varepsilon_{1}}{\varepsilon_{2}^{\prime}}
$$




$$
R \approx\left[\frac{\left(\varepsilon_{1}-\varepsilon_{2}^{\prime}\right)}{\left(\varepsilon_{1}+\varepsilon_{2}^{\prime}\right)}\right]^{2}=\left[\frac{\left(\varepsilon_{1} / \varepsilon_{2}^{\prime}-1\right)}{\left(\varepsilon_{1} / \varepsilon_{2}^{\prime}+1\right)}\right]^{2}=\left[\frac{\left(n_{1} /\left(n_{2}-1\right)\right.}{\left(n_{1} /\left(n_{2}+1\right)\right.}\right]^{2}=\left(\frac{n_{1}-n_{2}}{n_{1}+n_{2}}\right)^{2} .
$$

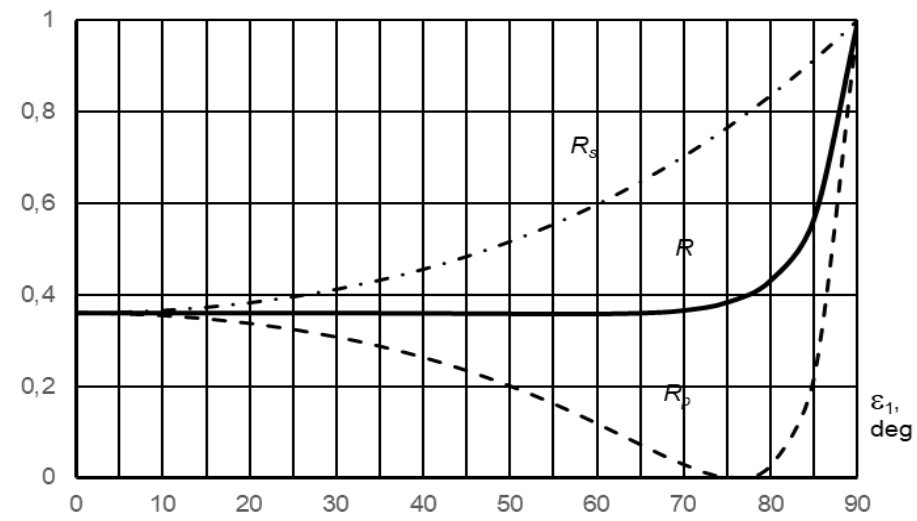

Figure 3 . The dependence of the total coefficient $R$ and the partial reflection coefficients $R_{p}$ and $R_{s}$ from the angle of incidence $\varepsilon_{1}$

If the angle of incidence $\varepsilon_{1}$ satisfies the condition $\varepsilon_{1}+\varepsilon_{2}^{\prime}=90^{\circ}$, then the partial reflection coefficient $R_{p}$ is zero. This means that the beam is polarized in the plane of incidence. It is not reflected and is completely refracted. In this case, the reflected beam will be fully polarized in a plane perpendicular to the plane of incidence. From the refraction law (4) we find the angle of incidence $\varepsilon_{1}=\varepsilon_{B}$, at which the reflected ray will be fully polarized

$$
\frac{n_{2}}{n_{1}}=\frac{\sin \varepsilon_{B}}{\sin \left(\pi / 2-\varepsilon_{B}\right)}=\operatorname{tg} \varepsilon_{B} .
$$

For germanium plate the Brewster angle is $\varepsilon_{B}=\operatorname{arctg} 4=76^{\circ}$ (Figure 3).

Let's determine the plate reflection coefficient when the angle of incidence is equal to the Brewster angle $\varepsilon_{B}$. From (3) we have

$$
R=\frac{1}{2} R_{s}=\frac{1}{2} \sin ^{2}\left(\varepsilon_{1}-\varepsilon_{2}^{\prime}\right)=\frac{1}{2} \sin ^{2}\left(2 \varepsilon_{B}-90^{\circ}\right) .
$$

For germanium plates the reflection coefficient is

$$
R=\frac{1}{2} \sin ^{2}\left(2 \cdot 76-90^{\circ}\right)=0.39 .
$$

Double-refractive polarizers have a transmittance of less than $50 \%$. Thus, the use of a germanium plate makes it possible to create highly efficient polarizers for the far infrared region of the spectrum ${ }^{14,15}$.

\section{METHOD FOR MEASURING POLARIZATION DEGREE USING GERMANIUM PLATE}

The above mentioned parameters of polarization are valid when a parallel beam of rays falls on the plate surface strictly at the Brewster angle $\varepsilon_{B}$. If the angle of incidence $\varepsilon_{1}$ differs from the Brewster angle, then the partial reflection coefficient $R_{p}$ will not be zero. This will cause the reflected radiation to be partially polarized.

On the basis of polarization degree definition ${ }^{3,4}$ let's determine degree of polarization of the reflected radiation at arbitrary angles of beam incidence $\varepsilon_{1}$

$$
P=\frac{I_{\max }-I_{\min }}{I_{\max }+I_{\min }}=\frac{I_{R_{s}}-I_{R p}}{I_{R s}+I_{R p}}=\frac{R_{s}-R_{p}}{R_{s}+R_{p}} .
$$


Using the Fresnel formula (3) and the refractive law (4), we obtain the dependence of the reflected radiation polarization degree from the incidence angle $\varepsilon_{1}$ which graphical representation is shown in Figure 4. It's analysis shows that:

1. Reflected radiation will be fully polarized $(P=100 \%)$ at an angle of incidence $\varepsilon_{1}=76^{\circ}$, which is equal to the Brewster angle.

2. At angles of incidence $\varepsilon_{1} \rightarrow 0^{\circ}$ and $\varepsilon_{1} \rightarrow 90^{\circ}$ the reflected radiation will be unpolarized $(P=0)$.

3. The degree of polarization decreases twice when the angle of incidence is $\varepsilon_{11}=54^{\circ}$ or $\varepsilon_{12}=86^{\circ}$.

4. Based on the results obtained, it is proposed to evaluate polarizers performance by half-width

$$
\Delta \varepsilon_{1}=\varepsilon_{12}-\varepsilon_{11} .
$$

For germanium plate we get $\Delta \varepsilon_{1}=32^{\circ}$.

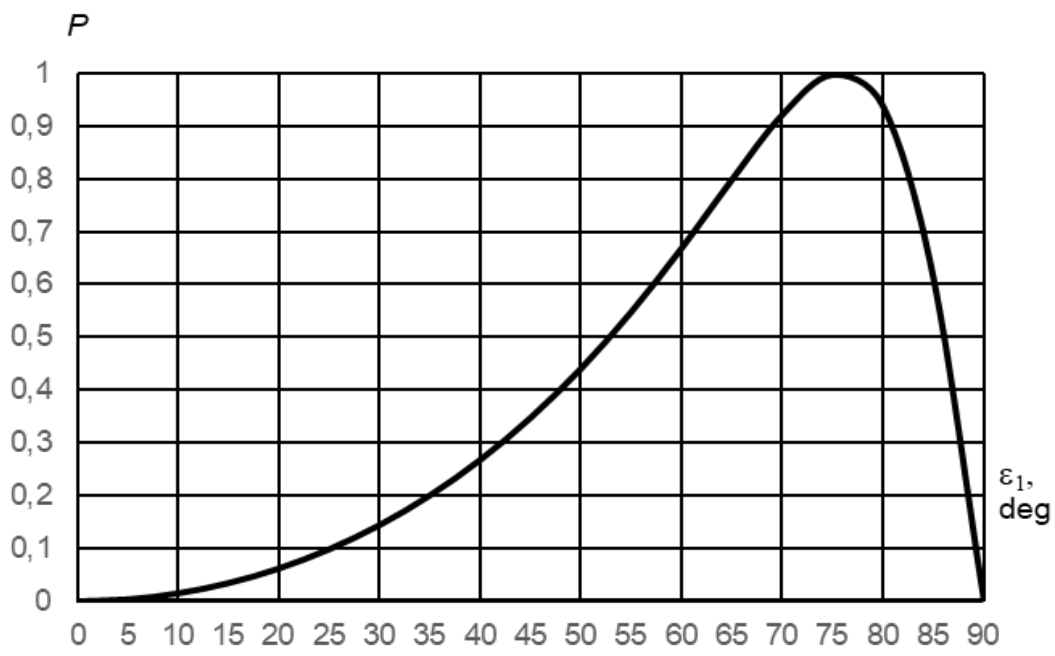

Figure 4. Dependence of reflected ray polarization degree on the angle of incidence $\varepsilon_{1}$

The main disadvantage of such polarizers is the deviation of optical axis at an angle $\alpha=2 \varepsilon_{B}=152^{\circ}$. This significantly complicates the opto-mechanical system of the polarization measuring device. To simplify the optical system of such devices, it is proposed to use a plane-parallel transmission germanium plate. Parallel beam of rays falls on the surface of the plate at the Brewster angle (Figure 5). Let's find the transmittance of such a plate and the polarization degree of radiation at the exit of the plate.

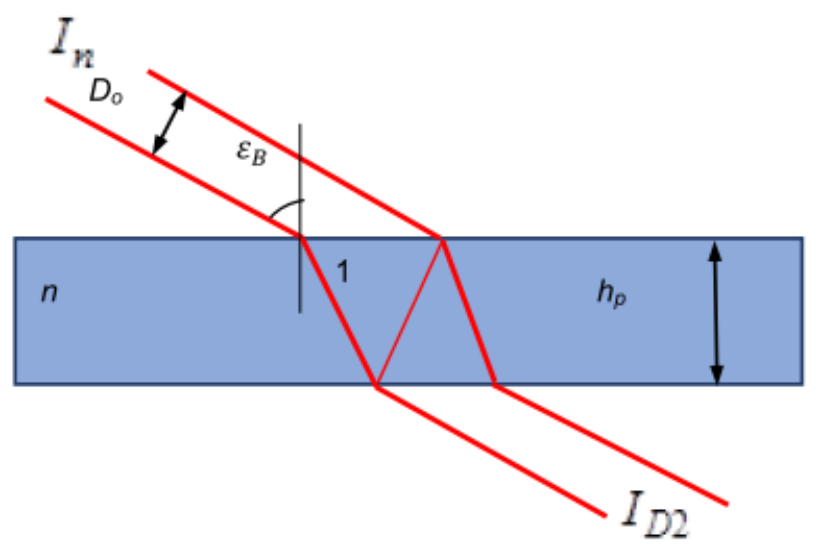

Figure 5. Transmission polarizer diagram 
Transmittance will be defined as $T=I_{D 2} / I_{n}$, where $I_{D 2}$ is radiation intensity at the exit of the plate. To find this intensity, let's consider a natural light model in the form of the sum of two waves, which are plane polarized in two mutually perpendicular planes (Figure 2). The energy transmittance of the plate surface for these waves is

$$
T_{p}=I_{D 1 p} / I_{n p}=1, T_{s}=I_{D 1 s} / I_{n s}=1-R_{s}=1-\sin ^{2}\left(2 \varepsilon_{B}-\varepsilon_{1}\right)=1-0.78=0.22 \text {. }
$$

The total energy transmittance is:

$$
T=\frac{I_{D 2}}{I_{n}}=\frac{I_{D p 2}+I_{D s 2}}{I_{n}}=\frac{1}{2}\left[\left(1-R_{p}\right)^{2}+\left(1-R_{s}\right)^{2}\right] .
$$

For germanium plates we have $T=\frac{1}{2}\left[1+(1-0.78)^{2}\right]=0.524$.

The degree of radiation polarization at the outlet of the plate is defined as

$$
P=\frac{I_{D p 2}-I_{D s 2}}{I_{D p 2}+I_{D s 2}}=\frac{\left(1-R_{p}\right)^{2}-\left(1-R_{s}\right)^{2}}{\left(1-R_{p}\right)^{2}+\left(1-R_{s}\right)^{2}} .
$$

For germanium plates we have $P=\frac{1-(1-0.78)^{2}}{1+(1-0.78)^{2}}=0.908$.

Let's determine the radiation polarization degree (11) at the exit of the plate at arbitrary angles of incidence $\varepsilon_{1}$, using the Fresnel formula (3) and the refraction law (4). After the corresponding transformations we obtain a dependence $P\left(\varepsilon_{1}\right)$ which graphical representation is shown in Figure 6. Its analysis leads to the following conclusions:

1. Reflected radiation will be fully polarized $(P=100 \%)$ at the angle of incidence $\varepsilon_{1} \rightarrow 90^{\circ}$. At angles of incidence $\varepsilon_{1} \rightarrow 0^{\circ}$ the radiation passing through the plate will be unpolarized $(P=0)$.

2. The degree of polarization decreases twice compared to the polarization degree at the Brewster angle, when the angle of incidence is $\varepsilon_{11}=50^{\circ}$.

3. Based on the obtained results, the polarizer efficiency is determined by the half-width (10) $\Delta \varepsilon_{1}=\varepsilon_{12}-\varepsilon_{11}=40^{\circ}$.

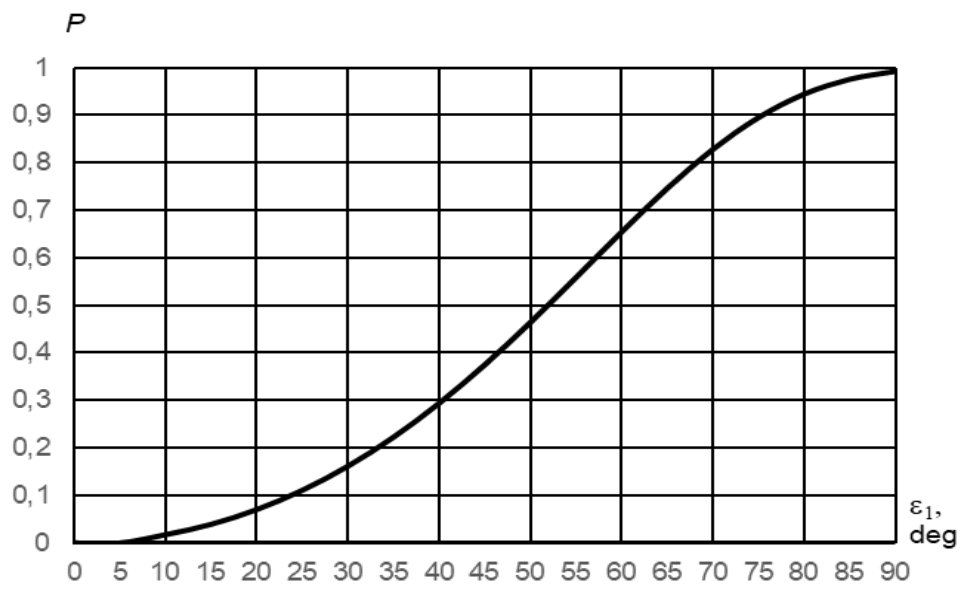

Figure 6. Dependence of polarization degree of the beam at the exit of germanium plate on the angle of incidence $\varepsilon_{1}$

The analysis of Figure 5 shows that the diameter $D_{o}$ of the input beam is limited by the thickness $h_{p}$ of the plate. The edge rays 1 and 2 of the input beam pass through the plate and form a beam of rays parallel to the input beam. As a result of repeated reflection of rays from the plate surfaces, several parallel beams of low intensity are formed at the output. The thickness $h_{p}$ of the plate is chosen on the condition that these beams do not overlap. To remove additional beams, you can set a field diaphragm after the plate. Diameter of field diaphragm is equal to the diameter $D_{o}$ of the input beam. Therefore, the main disadvantage of such a polarizer will be usage of considerable thick germanium plate to ensure the polarization of the input radiation of a given diameter. 


\section{CONCLUSIONS}

1. Application of Brewster law in reflecting radiation from a germanium plate allows to create high-efficiency polarizers for the infrared radiation of $8-14 \mu \mathrm{m}$ band. At an angle of incidence $\varepsilon_{1}=76^{\circ}$ the reflection coefficient of plate is 0.39 .

2. The study of the polarization degree of the reflected radiation showed that

- Radiation will be fully polarized at an incidence angle of $76^{\circ}$.

- The polarization degree decreases twice at angles of light incidence $\varepsilon_{11}=54^{\circ}$ and $\varepsilon_{12}=86^{\circ}$.

3. It is suggested to evaluate the polarizer's efficiency by the reflection coefficient when the angle of incidence is equal to the Brewster angle, and by the range of angles of incidence at which the reflected radiation polarization degree will be greater than $50 \%$.

4. The transmitting polarizer will be more efficient, since it does not deviate the optical axis by a considerable angle. The optical axis only gets slight parallel displacement. Such a polarizer provides a polarization degree of $90 \%$ and transmittance of about $50 \%$.

\section{REFERENCES}

[1] Pye, D., [Polarised Light in Science and Nature], IOP Publishing Ltd, USA (2001).

[2] Kolobrodov, V. G., Tymchyk, G. S., Mykytenko, V. I. and Kolobrodov, M. S., "Physical and mathematical model of the digital coherent optical spectrum analyzer," Optica Applicata 47(2), 273-282 (2017).

[3] Kolobrodov, V. G., Tymchyk, G. S., Kolobrodov, M. S., Vasyura, A. S., Komada, P. and Azeshova, Z., "The output signal of a digital optoelectronic processor," Proc. SPIE 10808, 108080W (2018).

[4] Tymchik, G. S., Skytsiouk, V. I., Klotchko, T. R., Popiel, P. and Begaliyeva, K., "The active surface of the sensor at a contact to the technological object," Proc. SPIE 10808, 108085G (2018).

[5] Ghatak, A., [Optics], The McGraw-Hill Companies, USA (2010).

[6] Suresh, G., [Wave optics], PHI Learning Private Limited, New Delhi (2012).

[7] Yan Zhang, Zhi-Guang Shi, and Tiao-Wen Qiu, "Infrared small target detection method based on decomposition of polarization information," Journal of Electronic Imaging 26(3), 033004-1 - 033004-7 (2017).

[8] Vollmer, M., Henke, S., "Kar Identification and Suppression of Thermal Reflections in Infrared Thermal Imaging," Proc. InfraMation 2004, INC 104 A (2004).

[9] Tymchyk, G. S., Skytsiouk, V. I., Klotchko, T. R., Ławicki, T. and Denissova, N., "Distortion of geometric elements in the transition from the imaginary to the real coordinate system of technological equipment," Proc. SPIE 10808, 108085C (2018).

[10] Tymchyk, G. S., Skytsyuk, V. I., Klotchko, T. R., Bezsmertna, H., Wójcik, W., Luganskaya, S., Orazbekov, Z. and Iskakova, A., "Diagnosis abnormalities of limb movement in disorders of the nervous system," Proc. SPIE 10445, 104453S (2017).

[11] Sadjadi, F. A. and Chun, C. S. L., "New experiments in the use of infrared polarization in the detection of small targets," Proc. SPIE 4379 (2001).

[12] W. Wojcik, S. Cakala, A. Kotyra et al., "Analysis of the operation of an electrooptical Pockels effect sensor," Technology and Applications of Light Guides, 3189, 110-121 (1997)..

[13] Pavlov, S. V., Kozhemiako, V. P., Kolesnik, P. F., et al., [Physical principles of biomedical optics: monograph], VNTU, Vinnytsya, 1-152 (2010).

[14] Pavlov, S. V., Tuzhanskyy, S. E., Kozlovska, T. I. and Kozak, A.V., "A simulation model of distribution of optical radiation in biological tissues," Visnyk VNTU 3, 191-195 (2011).

[15] Rovira, R. H., Tuzhanskyy, S. Ye., Pavlov, S. V., et al., "Polarimetric characterisation of histological section of skin with pathological changes," Proc. SPIE 10031, 100313E (2016). 